\title{
Cross-sectional analysis of immunosuppressive regimens focused on everolimus after liver transplantation in a Korean high-volume transplantation center
}

\author{
Sang-Hyun Kang1, Shin Hwang', Tae-Yong Ha', Gi-Won Song', Dong-Hwan Jung', Chul-Soo Ahn', \\ Deok-Bog Moon ${ }^{1}$, Ki-Hun Kim ${ }^{1}$, Gil-Chun Park', Young-In Yoon ${ }^{1}$, Yo-Han Park ${ }^{2}$, Hui-Dong Cho', \\ Jae-Hyun Kwon ${ }^{1}$, Yong-Kyu Chung ${ }^{1}$, Jin Uk Choi ${ }^{1}$, Sung-Gyu Lee ${ }^{1}$ \\ 'Division of Liver Transplantation and Hepatobiliary Surgery, Department of Surgery, Asan Medical Center, \\ University of Ulsan College of Medicine, Seoul, Korea; \\ ${ }^{2}$ Department of Surgery, Busan Paik Hospital, Inje University College of Medicine, Busan, Korea
}

Background: The mammalian target of the rapamycin inhibitor has dual inhibitory effects on cell growth and angiogenesis. This study aimed to analyze the usage of everolimus on actual immunosuppression (IS) regimens through a cross-sectional study in a high-volume liver transplantation (LT) center.

Methods: Our institutional LT database was searched for adult patients who underwent primary LT surgery between January 2010 and December 2016. We identified 2,093 LT recipients with observation periods of 1 to 8 years.

Results: We divided the 2,093 recipients into three groups according to the posttransplant follow-up period as follows: group A (12-36 months; $n=680)$, group B (37-60 months; $n=560)$, and group $C(>60$ months; $n=853)$. The individual IS agents were tacrolimus in 1,807 patients $(86.3 \%)$, cyclosporine in 169 patients $(8.1 \%)$, mycophenolate mofetil (MMF) in 1,310 patients $(62.6 \%)$, and everolimus in 115 patients $(5.5 \%)$. The most common IS regimens were tacrolimus-MMF combination and tacrolimus monotherapy, regardless of the posttransplant period. Patients with pretransplant malignancies were administered everolimus more frequently than those without pretransplant malignancies $(\mathrm{P}<0.001)$. In 102 patients with hepatocellular carcinoma recurrence or de novo malignancies, IS regimens included everolimus-tacrolimus in 41 patients $(40.2 \%)$, tacrolimus-MMF in 27 patients $(26.4 \%)$, tacrolimus in 20 patients $(19.6 \%)$, MMF in 10 patients (9.8\%), cyclosporine in three patients $(2.9 \%)$, and cyclosporine-MMF in one patient $(1.0 \%)$.

Conclusions: Administration of everolimus after LT has been gradually increasing with the expansion of indications in our institutional practice. Currently, the role of everolimus is minimal and not comparable to that of tacrolimus, but it has a unique position in the field of IS after LT.

Keywords: Tacrolimus; Mycophenolate mofetil; Everolimus; Malignancy; Hepatocellular carcinoma

\section{INTRODUCTION}

Received August 7, 2019

Revised September 16, 2019

Accepted October 11, 2019

Correspondence to: Shin Hwang

Department of Surgery, Asan Medical Center, University of Ulsan

College of Medicine, 88 Olympic-ro 43-gil, Songpa-gu, Seoul 05505, Korea

Tel: +82-2-3010-3930, Fax: +82-2-3010-6701

E-mail: shwang@amc.seoul.kr
Immunosuppression (IS) is essential after liver transplantation (LT), with the exception of the development of operational tolerance. To date, every available immunosuppressive agent (ISA) has been administered after LT. Most IS protocols for LT include calcineurin inhibitors (CNI), mycophenolate mofetil (MMF), and steroids [1-3]. Steroids are usually tapered off early or in-

This is an Open Access article distributed under the terms of the Creative Commons Attribution Non-Commercial License (http://creativecommons. org/ licenses/by-nc/4.0/) which permits unrestricted non-commercial use, distribution, and reproduction in any medium, provided the original work is properly cited. 


\section{HIGHLIGHTS}

- A cross-sectional study in a high-volume center revealed that the most common immunosuppressive regimens were tacrolimus-mycophenolate mofetil combination and tacrolimus monotherapy.

- Everolimus use has been gradually increasing with expansion of indications in our institutional practice.

tentionally omitted after LT due to the relatively low incidence of acute rejection and high proportion of concurrent hepatocellular carcinomas (HCCs). For the CNIsparing effect, MMF has been used concurrently or alone.

The mammalian target of rapamycin inhibitor (mTORi) is an ISA that interrupts the interleukin-2 proliferative response cascade and has dual inhibitory effects on cell growth and angiogenesis. mTORi is increasingly administered as indicated for renal dysfunction or malignancies. Everolimus has been financially covered by the Korean social health insurance since early 2016 and is reported to be beneficial in reducing the risk of HCC recurrence after LT as well as being effective after overt HCC recurrence [4-6]. However, the real-world status of everolimus administration after LT in Korea has not been well reported $[1,3,5,7,8]$. The purpose of this study was to analyze the use of everolimus on actual IS regimens through a cross-sectional study in a Korean high- volume LT center.

\section{METHODS}

\section{Study Design and Patient Selection}

This cross-sectional study analyzed actual ISA regimens in adult LT recipients, with a focus on everolimus. We set the study period for cross-sectional review as 3 months from January 2018 to March 2018. The LT database at our institution was searched to identify adult patients who underwent primary LT during between January 2010 and December 2016. The inclusion criteria were patient survival for at least 12 months following LT and until the end of December 2018, recipient age $\geq 18$ years at LT surgery, and regular visits to our institution's outpatient clinic. Finally, we identified 2, 093 LT recipients with an observation period of between 1 to 8 years. Our study protocol was approved by the Institutional Review Board of our institution.

\section{Institutional IS Regimen Protocols}

Primary IS protocols used for adult LT recipients at our institution consisted of interleukin-2 receptor inhibitor, intraoperative steroid bolus $(5-10 \mathrm{mg} / \mathrm{kg}$ ), intravenous or oral $\mathrm{CNI}$ and corticosteroid recycling beginning on day 1 , and adjunctive MMF for patients showing CNI-associated adverse effects or for IS augmentation. For CNI-sparing to control CNI-associated adverse effects, tacrolimus and cyclosporine were occasionally exchanged. There was no differences in IS regimens between living-donor and deceased-donor LTs. Corticosteroids were rapidly tapered off within the first 3 months post LT $[3,9,10]$.

With regards to mTORi, we currently use only everolimus in our institution as it is financially covered for LT recipients by the Korean social health insurance program. The main indications for everolimus at our institution include HCC recurrence, de novo malignancy, and renal dysfunction. Intentional weaning from all ISAs is, to date, not considered at our institution, except for in cases of life-threatening severe infection or terminal status due to advanced malignancies. As everolimus was not included as a primary ISA at our institution during the study period, it was not commonly used prior to HCC recurrence or the development of de novo malignancies. If recurrence of $\mathrm{HCC}$ occurred, the IS regimen was switched towards everolimus-based regimens if the adverse side effects were tolerable.

\section{Stratification of LT Recipients According to Posttransplant Periods}

As the peritransplant condition of recipients was diverse, the IS regimens were also highly variable. Thus, we did not include IS regimens during the first 1 year in the present analysis.

We divided 2,093 recipients into three groups according to the posttransplant follow-up period, as follows: 2nd and 3rd years (12-36 months, group $A: n=680)$, 4th and 5th years $(37-60$ months, group $B: n=560)$ and beyond the 5th year ( $>60$ months, group $C: n=853$ ). These 
patients were also further divided according to the presence of liver malignancies at the point LT surgery as well as development of de novo malignancies following LT. Primary liver malignancies included HCC and other incidentally detected rare liver malignancies in the explant livers such as combined HCC-cholangiocarcinoma, intrahepatic cholangiocarcinoma, and neuroendocrine tumors.

\section{Statistical Analysis}

Continuous variables are reported as means with standard deviations. Categorical variables were compared using the chi-square test. A P-value of $<0.05$ was considered to be statistically significant. All statistical analyses were performed using IBM SPSS ver. 22.0 (IBM Corp., Armonk, NY, USA).

\section{RESULTS}

\section{Stratification of Patients According to Posttransplant Periods}

The numbers of LT recipients according to the posttransplant periods were $680(32.5 \%)$ in group A, 560 $(26.8 \%)$ in group B and $853(40.1 \%)$ in group C. The clinical profiles are summarized in Table 1. Pretransplant malignancies were diagnosed in 980 patients (46.8\%).

\section{Patterns of IS Regimens According to Posttransplant Periods}

Individual ISAs used in all patients were tacrolimus in 1,807 patients (86.3\%), cyclosporine in 169 patients (8.1\%), MMF in 1,310 patients $(62.6 \%)$, and everolimus in 115 patients $(5.5 \%)$. In group A, the ISA regimens included tacrolimus in 652 patients $(95.9 \%)$, cyclosporine in 18 patients (2.6\%), MMF in 387 patients (56.9\%), and everolimus in 64 patients $(9.4 \%)$. The most common IS regimens were tacrolimus-MMF combination $(n=358$,

Table 1. Comparison of recipient profiles according to the posttransplant periods

\begin{tabular}{|c|c|c|c|c|}
\hline \multirow{2}{*}{ Variable } & \multicolumn{3}{|c|}{ Recipient group } & \multirow{2}{*}{ Total } \\
\hline & Group A (12-36 mon) & Group B (37-60 mon) & Group C (>60 mon $)$ & \\
\hline No. of cases & $680(32.5)$ & $560(26.8)$ & $853(40.1)$ & 2,093 \\
\hline Age at LT (yr) & $53.3 \pm 9.1$ & $52.4 \pm 9.1$ & $51.6 \pm 8.3$ & $52.3 \pm 8.7$ \\
\hline Age at study (yr) & $55.8 \pm 9.0$ & $59.3 \pm 9.0$ & $58.7 \pm 8.2$ & $57.1 \pm 8.7$ \\
\hline \multicolumn{5}{|l|}{ Sex } \\
\hline Male & $500(73.5)$ & $409(73.0)$ & $648(76.1)$ & $1,556(74.4)$ \\
\hline Female & $180(26.5)$ & $151(27.0)$ & $204(23.9)$ & $536(25.6)$ \\
\hline \multicolumn{5}{|l|}{ Primary liver disease } \\
\hline Hepatitis B & $357(52.5)$ & $329(60.5)$ & $570(66.8)$ & $1,266(60.5)$ \\
\hline Hepatitis C & $27(4.0)$ & $39(7.0)$ & $51(6.0)$ & $117(5.6)$ \\
\hline Alcoholic disease & $179(26.3)$ & $107(19.1)$ & $118(17.9)$ & $404(19.4)$ \\
\hline Others & $117(17.2)$ & $75(13.4)$ & $114(13.4)$ & $306(14.6)$ \\
\hline \multicolumn{5}{|l|}{ Type of LT (n) } \\
\hline Deceased-donor & $94(13.8)$ & $58(10.4)$ & $108(12.7)$ & $260(12.4)$ \\
\hline Living-donor & $586(86.2)$ & $502(89.6)$ & $745(87.3)$ & $1833(87.6)$ \\
\hline \multicolumn{5}{|c|}{ Pretransplant malignancy (n) } \\
\hline Present & $295(43.4)$ & $262(46.8)$ & $423(49.6)$ & $980(46.8)$ \\
\hline Absent & $385(56.6)$ & $298(53.2)$ & $430(50.4)$ & $1,113(53.2)$ \\
\hline \multicolumn{5}{|c|}{ De novo malignancy ( $\mathrm{n}$ ) } \\
\hline Present & $8(1.2)$ & $24(4.3)$ & $37(4.3)$ & $69(3.3)$ \\
\hline Absent & $672(98.8)$ & $536(95.7)$ & $816(95.7)$ & $2,024(96.7)$ \\
\hline
\end{tabular}

Values are presented as number $(\%)$ or mean \pm standard deviation.

LT, liver transplantation. 
$52.6 \%)$ and tacrolimus monotherapy ( $\mathrm{n}=230,33.8 \%)$. In group B, the ISA regimens included tacrolimus in $491 \mathrm{pa}-$ tients $(87.7 \%)$, cyclosporine in 46 patients $(8.2 \%)$, MMF in 378 patients $(67.5 \%)$, and everolimus in $27 \mathrm{pa}-$ tients $(4.8 \%)$. The most common IS regimens were tacrolimus-MMF combination $(\mathrm{n}=328,58.6 \%)$ and tacrolimus monotherapy $(n=137,24.5 \%)$. In group $C$, the ISA regimens included tacrolimus in 664 patients (77.8\%), cyclosporine in 105 patients (12.3\%), MMF in 545 patients $(63.9 \%)$, and everolimus in 24 patients $(2.8 \%)$ recipients. The most common IS regimens were tacrolimus-MMF combination $(n=408,47.8 \%)$ and tacrolimus monotherapy $(n=232,27.2 \%)$. The patterns of ISA use are collectively depicted in Fig. 1. The proportion of recipients receiving everolimus gradually increased over time with the highest proportion occurring in the most recent $\mathrm{LT}$ cases, as shown by administration rates of $9.4 \%, 4.8 \%$, and $2.8 \%$ in groups $\mathrm{A}, \mathrm{B}$ and $\mathrm{C}$, respectively.

\section{Patterns of IS Regimens According to Pretransplant Malignancy}

In the 1,113 patients without pretransplant malignancies, the ISA regimens included tacrolimus in 962 patients (86.4\%), cyclosporine in 86 patients $(7.7 \%)$, MMF in 699 patients $(62.8 \%)$, and everolimus in 28 patients $(2.5 \%)$. The most common IS regimens were tacrolimus-MMF combination $(n=585,52.6 \%)$ and tacrolimus monotherapy ( $\mathrm{n}=349,31.4 \%$ ). In the 980 patients with pretransplant malignancies, the ISA regimens included tacrolimus in 845 patients $(86.2 \%)$, cyclosporine in 83 patients $(8.5 \%)$, MMF in 611 patients $(62.3 \%)$, and everolimus in 87 patients $(8.9 \%)$. The most common IS regimens were tacrolimus-MMF combination $(n=509$, $51.9 \%)$ and tacrolimus monotherapy $(n=250,25.5 \%)$ (Fig. 2). There was no difference in ISA use between the two groups, with the exception of everolimus ( $P$ $<0.001)$. The proportion of recipients who received everolimus was $8.9 \%$ and $2.5 \%$ in recipients with and without pretransplant malignancies, respectively.
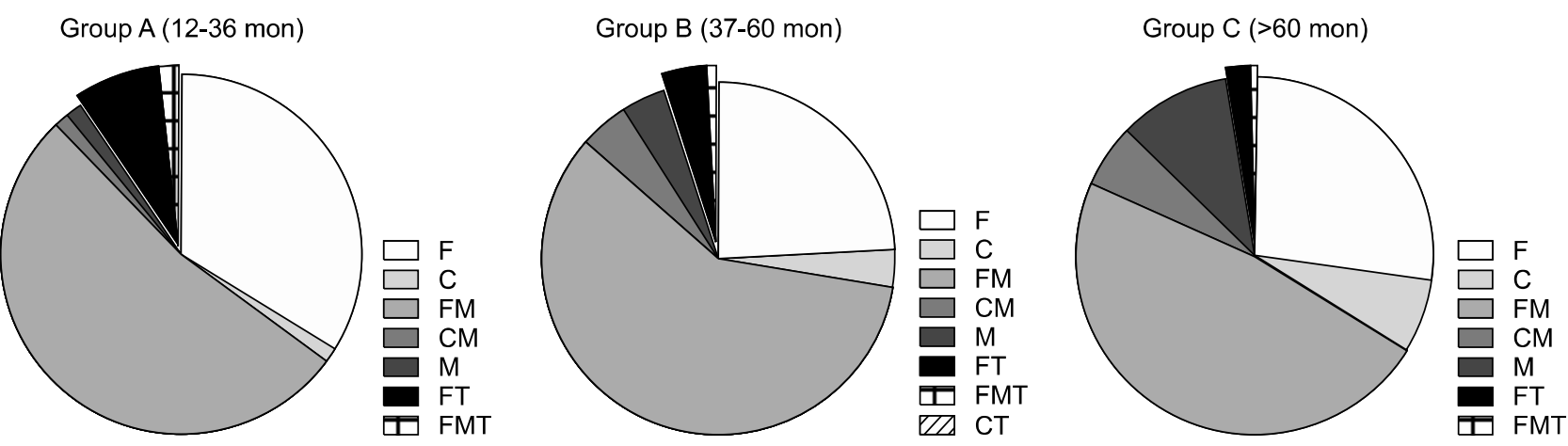

Fig. 1. Proportion of immunosuppressive regimens in 2,093 liver transplant recipients according to posttransplant periods. F, tacrolimus $\mathrm{C}$, cyclosporine; M, mycophenolate mofetil; $\mathrm{T}$, everolimus. Letters indicate the combination therapy.
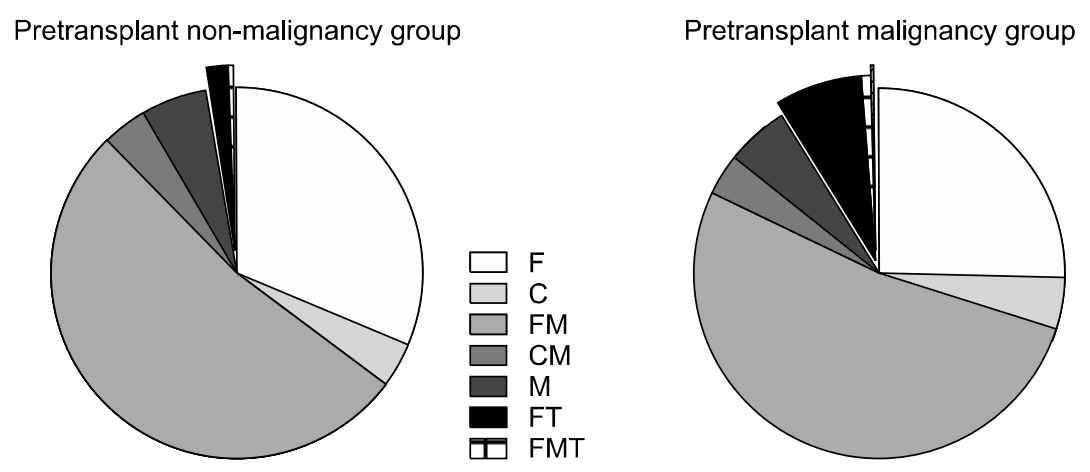

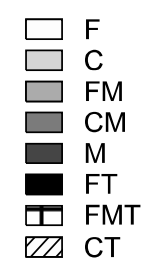

Fig. 2. Proportion of immunosuppressive regimens in liver transplant recipients with or without pretransplantation liver malignancies. $\mathrm{F}$, tacrolimus; C, cyclosporine; $\mathrm{M}$, mycophenolate mofetil; $\mathrm{T}$, everolimus. Letters indicate the combination therapy. 


\section{Patterns of IS Regimens According to Posttransplant Malignancy}

At the end of study period, the number of living patients with HCC recurrence or de novo malignancies were 52 and 67 , respectively. With exclusion of the overlapped patients $(n=17)$, a total of 102 patients were categorized as the posttransplant malignancy group. The IS regimens included everolimus-tacrolimus combination in $41 \mathrm{pa}-$ tients $(40.2 \%)$, tacrolimus-MMF combination in $27 \mathrm{pa}-$ tients $(26.4 \%)$, tacrolimus monotherapy in 20 patients (19.6\%), MMF monotherapy in 10 patients $(9.8 \%)$, cyclosporine monotherapy in three patients $(2.9 \%)$, and cyclosporine-MMF combination in one patient $(1.0 \%)$. Thus, 41 recipients $(40.2 \%)$ were switched to everolimus due to HCC recurrence or the development of de novo malignancies [5]. Of the 115 patients treated with everolimus, 74 patients (64.3\%) were administered everolimus to prevent HCC recurrence and de novo malignancy development or to inducing CNI-sparing effect.

\section{Institutional Experience of Switching to mTORi in Recipients with HCC Recurrence}

Of the 232 recipients who showed posttransplant HCC recurrence prior to December 2015, mTORi was administered to 51 recipients. However, 12 recipients (23.5\%) dropped out within first 3 months due to adverse effects or other causes, including excessive financial burdens. However, subgroup analysis revealed that mTORi administration was stopped in only four of these patients as the severe adverse effects were not controlled despite dosage reduction. In the remaining 39 patients, mTORi was maintained to date or until patient death. After the implementation of insurance coverage for everolimus in Korea, the dropout rate of everolimus was lowered to two out of 30 recipients $(6.7 \%)$. Everolimus was used alone or in combination with minimal CNI or MMF. None of the everolimus recipients experienced overt acute rejection that required high-dose steroid therapy [5].

\section{DISCUSSION}

The recent introduction of $\mathrm{mTOR}$ inhibitors in Korea has resulted in noticeable changes to IS regimens. Everolimus coverage was introduced to Korean social health insurance in early 2016. Thereafter, the rate at which mTORi administration was terminated decreased significantly. So far, we do not consider this agent to be a primary ISA, thus it is not, as of yet, frequently used in our institution. Everolimus can be administered following the first 1 month post LT, primarily due to the risk of hepatic artery thrombosis and issues with regards to wound healing. Korean social health insurance policy forcefully suggests administrating tacrolimus and everolimus concurrently.

In our institution, the first indication that everolimus should be prescribed is HCC recurrence or the development of de novo malignancies. This is due to the fact that mTORi are known to be the only ISA with anti-tumor effects [11-16]. Our study revealed that the overall use of everolimus is not high, but its use is gradually increasing as the criteria for its use are expanded. Due to the social health insurance policy and its relatively low immunosuppressive potency, it is usually administered concurrently with tacrolimus or MMF. Everolimus monotherapy has rarely been adopted, especially in patients with overt presentation of HCC recurrence or de novo malignancies. We intend to concurrently use mTORi and sorafenib with the expectation of the synergistic effect against HCC recurrence. However, their beneficial effects were not demonstrated in our precedent clinical study [5].

The second indication for everolimus use in our institution is renal dysfunction because of its CNI-sparing effect. It is known to be beneficial in effectively decreasing the concentration of CNIs. The H2304 study is a multicenter, open-label, randomized control study to evaluate the efficacy and safety of everolimus to eliminate or reduce tacrolimus in de novo LT recipients $[6,17,18]$. This study showed clear separation and clinically relevant reductions in tacrolimus exposure in the everolimus-reduced tacrolimus group, which resulted in improvements in renal function. However, the everolimus monotherapy following tacrolimus group was stopped early due to a high incidence of acute rejection. It is a critically important finding that everolimus is not sufficiently potent to pre- 
vent acute rejection, especially during the first posttransplant year.

In the current Korean setting, CNI-sparing effect of everolimus does not appear critical because many Korean LT centers accumulated experience on reduced CNI-MMF combination or MMF monotherapy to cope with $\mathrm{CNI}-$ induced renal dysfunction [3,9,10]. At this point, we emphasize that MMF dosing requires mycophenolic acid (MPA) therapeutic drug monitoring because a considerable proportion of LT recipients are poor absorbers of MMF. Poor absorption of MMF is not an eligible indication for MMF monotherapy. We arbitrarily defined poor MMF absorption as a 12 hour trough level of MPA of $<1.0 \mathrm{mg} / \mathrm{L}$ after administration of MMF $500 \mathrm{mg}$ twice per day [9]. Since the potency of MMF for IS is not sufficiently high [2], MMF monotherapy was rarely used within the first year of LT at our institution. After introduction of everolimus, a triple combination of $\mathrm{MMF}$ and everolimus with very low-dose tacrolimus was selectively used in recipients showing progressive renal dysfunction during the first year post LT [19-22]. Considering that most of these recipients undergoing MMF monotherapy had renal dysfunction, meticulous adjustment of MMF dosage is necessary because renal dysfunction interferes with metabolism and excretion of MPA [1-3,9,10].

The third indication for everolimus use in our institution is refractory rejection. The primary indications for the use of mTORi after LT are reported, in decreasing order, as follows: renal failure, prevention of HCC recurrence, recurrent HCC, de novo malignancies, diabetes, hypertension, neurotoxicity, and others [23]. However, the common indications for switching to everolimus are reported to be refractory rejection, extended HCC, de novo malignancies, severe neurotoxicity, HCC recurrence, renal insufficiency, and others [24]. After we experienced a few cases showing marked therapeutic effect on refractory rejection, we have carefully added everolimus to tacrolimus in patients presenting with unexplained hepatic dysfunction.

Everolimus can induce various adverse side-effects. In the H2304 study, the incidence of severe adverse side-effects was $29.7 \%$ in the everolimus-reduced ta- crolimus group, which is higher than the $21.5 \%$ reported in the tacrolimus control group [18]. There are some treatment algorithms for adverse side-effects. For oral ulcers and skin lesions, the initial treatment is local control; if clinical improvement is not achieved, dosage should be reduced and if the presentation is serious, drug administration should be stopped. With regards to proteinuria, mild proteinuria $<1 \mathrm{~g} /$ day can be observed. If moderate proteinuria develops, everolimus should be reduced or stopped. For dyslipidemia, if low density lipoprotein cholesterol is elevated, statins can be used. For mild-to-moderate peripheral edema, the first step is support care. Additionally, early administration of everolimus can increase the risk of hepatic artery thrombosis, thus it is recommended that it is not prescribed until after the first month post LT surgery [17].

This study has several limitations. First, this is a retrospective single-center study with cross-sectional review covering a short period. Second, we did not separately present the profiles of patients after HCC recurrence or the development of de novo malignancies alive at the time of data collection, as these data will be presented in future reports of ongoing studies.

In conclusion, the rate of everolimus use is gradually increasing in our institution as the criteria for its use following LT are expanding. Currently, the role of everolimus is minimal and is not comparable to that of tacrolimus, but it has a unique position in the field of IS after LT.

\section{ACKNOWLEDGMENTS}

\section{Conflict of Interest}

No potential conflict of interest relevant to this article was reported.

\section{Funding/Support}

This study was supported by the intramural research fund of Asan Medical Center Organ Transplantation Center.

This study was supported by research grant from the Korean Society for Transplantation (2019-04-01001004). 


\section{ORCID}

Sang-Hyun Kang

Shin Hwang

Tae-Yong Ha

Gi-Won Song

Dong-Hwan Jung

Chul-Soo Ahn

Deok-Bog Moon

Ki-Hun Kim

Gil-Chun Park

Young-In Yoon

Yo-Han Park

Hui-Dong Cho

Jae-Hyun Kwon

Yong-Kyu Chung

Jin-Uk Choi

Sung-Gyu Lee https://orcid. org/0000-0002-8518-1941 https://orcid. org/0000-0002-9045-2531

https://orcid.org/0000-0001-9932-0212 https://orcid.org/0000-0002-4235-0434 https://orcid.org/0000-0001-5984-023X https://orcid.org/0000-0002-3844-3646 https://orcid.org/0000-0002-8209-3540 https://orcid.org/0000-0002-4016-0995 https://orcid.org/0000-0003-1631-3258 https://orcid.org/0000-0002-9308-0366 https://orcid.org/0000-0002-2242-0968 https://orcid.org/0000-0001-8501-3385 https://orcid.org/0000-0001-8605-9350 https://orcid.org/0000-0002-2132-2450 https://orcid.org/0000-0001-8078-0593 https://orcid.org/0000-0001-9161-3491

\section{Author Contributions}

Conceptualization: SH. Data curation: SH, SHK, YKC. Formal analysis: TYH, GWS, DHJ, CSA, DBM, KHK, GCP, YIY, YHP, HDC, JHK, JUC. Funding acquisition: SH. Methodology: SH, SHK. Project administration: SH, SGL. Visualization: SH. Writing - original draft: SH, SHK. Writing - review \& editing: SH.

\section{REFERENCES}

1. Kang SH, Hwang S, Ha TY, Song GW, Jung DH, Kim $\mathrm{KH}$, et al. Tailored long-term immunosuppressive regimen for adult liver transplant recipients with hepatocellular carcinoma. Korean J Hepatobiliary Pancreat Surg 2014;18:48-51.

2. Hwang S, Lee SG, Ahn CS, Kim KH, Moon DB, Ha TY, et al. A clinical assessment of mycophenolate drug monitoring after liver transplantation. Clin Transplant 2010;24:E35-42.

3. Hwang S, Ahn CS, Kim KH, Moon DB, Ha TY, Song $\mathrm{GW}$, et al. A cross-sectional analysis of long-term immunosuppressive regimens after liver transplantation at Asan Medical Center: increased preference for mycophenolate mofetil. Ann Hepatobiliary Pancreat Surg 2018; 22:19-26.

4. Clavien PA, Lesurtel M, Bossuyt PM, Gores GJ, Langer $\mathrm{B}$, Perrier A, et al. Recommendations for liver trans- plantation for hepatocellular carcinoma: an international consensus conference report. Lancet Oncol 2012;13: e11-22.

5. Jung DH, Tak E, Hwang S, Song GW, Ahn CS, Kim $\mathrm{KH}$, et al. Antitumor effect of sorafenib and mammalian target of rapamycin inhibitor in liver transplantation recipients with hepatocellular carcinoma recurrence. Liver Transpl 2018;24:932-45.

6. Fischer L, Saliba F, Kaiser GM, De Carlis L, Metselaar HJ, De Simone P, et al. Three-year outcomes in de novo liver transplant patients receiving everolimus with reduced tacrolimus: follow-up results from a randomized, multicenter study. Transplantation 2015;99:1455-62.

7. Alshahrani AA, Ha SM, Hwang S, Ahn CS, Kim KH, Moon DB, et al. Clinical features and surveillance of very late hepatocellular carcinoma recurrence after liver transplantation. Ann Transplant 2018;23:659-65.

8. Alshahrani AA, Hwang S, Song GW, Moon DB, Jung $\mathrm{DH}$, Ahn CS, et al. Management of very late peritoneal metastasis of hepatocellular carcinoma 10 years after liver transplantation: lessons from two cases. Ann Hepatobiliary Pancreat Surg 2018;22:136-43.

9. Park YH, Hwang S, Song GW, Jung DH, Ahn CS, Kim $\mathrm{KH}$, et al. Correlation between mycophenolic acid blood level and renal dysfunction in stable liver transplant recipients receiving mycophenolate monotherapy. Transplant Proc 2014;46:811-5.

10. Hwang S, Song GW, Jung DH, Park GC, Ahn CS, Moon $\mathrm{DB}$, et al. Intra-individual variability of mycophenolic acid concentration according to renal function in liver transplant recipients receiving mycophenolate monotherapy. Ann Hepatobiliary Pancreat Surg 2017;21:11-6.

11. Yamanaka K, Petrulionis M, Lin S, Gao C, Galli U, Richter $\mathrm{S}$, et al. Therapeutic potential and adverse events of everolimus for treatment of hepatocellular carcinoma: systematic review and meta-analysis. Cancer Med 2013; $2: 862-71$

12. Navarro-Villarán E, Tinoco J, Jiménez G, Pereira S, Wang J, Aliseda S, et al. Differential antitumoral properties and renal-associated tissue damage induced by tacrolimus and mammalian target of rapamycin inhibitors in hepatocarcinoma: in vitro and in vivo studies. PLoS One 2016;11:e0160979.

13. Zheng JF, Lu J, Wang XZ, Guo WH, Zhang JX. Comparative metabolomic profiling of hepatocellular carcinoma cells treated with sorafenib monotherapy vs. sorafenib-everolimus combination therapy. Med Sci Monit 2015;21: 1781-91.

14. Cholongitas E, Goulis I, Theocharidou E, Antoniadis N, 
Fouzas I, Giakoustidis D, et al. Everolimus-based immunosuppression in liver transplant recipients: a single-centre experience. Hepatol Int 2014;8:137-45.

15. Cholongitas E, Mamou C, Rodríguez-Castro KI, Burra P. Mammalian target of rapamycin inhibitors are associated with lower rates of hepatocellular carcinoma recurrence after liver transplantation: a systematic review. Transpl Int 2014;27:1039-49.

16. Gomez-Martin C, Bustamante J, Castroagudin JF, Salcedo M, Garralda E, Testillano M, et al. Efficacy and safety of sorafenib in combination with mammalian target of rapamycin inhibitors for recurrent hepatocellular carcinoma after liver transplantation. Liver Transpl 2012;18: 45-52.

17. De Simone P, Nevens F, De Carlis L, Metselaar HJ, Beckebaum S, Saliba F, et al. Everolimus with reduced tacrolimus improves renal function in de novo liver transplant recipients: a randomized controlled trial. Am J Transplant 2012;12:3008-20.

18. Saliba F, De Simone P, Nevens F, De Carlis L, Metselaar $\mathrm{HJ}$, Beckebaum S, et al. Renal function at two years in liver transplant patients receiving everolimus: results of a randomized, multicenter study. Am J Transplant 2013; 13:1734-45.

19. Jiménez-Pérez M, González Grande R, Rando Muñoz FJ, de la Cruz Lombardo J, Muñoz Suárez MA, Fernández Aguilar JL, et al. Everolimus plus mycophenolate mofetil as initial immunosuppression in liver transplantation. Transplant Proc 2015;47:90-2.

20. Bilbao I, Dopazo C, Castells L, Lazaro J, Caralt M, Sapisochin $\mathrm{G}$, et al. Immunosuppression based on everolimus in liver transplant recipients with severe early post-transplantation neurotoxicity. Transplant Proc 2014;46:3104-7.

21. Beckebaum S, Cicinnati VR, Radtke A, Kabar I. Calcineurin inhibitors in liver transplantation: still champions or threatened by serious competitors? Liver Int 2013;33:656-65.

22. Herzer K, Strassburg CP, Braun F, Engelmann C, Guba $\mathrm{M}$, Lehner F, et al. Selection and use of immunosuppressive therapies after liver transplantation: current German practice. Clin Transplant 2016;30:487-501.

23. Alegre C, Jiménez C, Manrique A, Abradelo M, Calvo J, Loinaz C, et al. Everolimus monotherapy or combined therapy in liver transplantation: indications and results. Transplant Proc 2013;45:1971-4.

24. Saliba F, Dharancy S, Lorho R, Conti F, Radenne S, Neau-Cransac M, et al. Conversion to everolimus in maintenance liver transplant patients: a multicenter, retrospective analysis. Liver Transpl 2011;17:905-13. 